\title{
Models of the formation of the planets in the 47 UMa system
}

\author{
K. Kornet ${ }^{1}$, P. Bodenheimer ${ }^{2}$, and M. Różyczka ${ }^{1}$ \\ 1 Nicolaus Copernicus Astronomical Center, Bartycka 18 , Warsaw, 00-716, Poland \\ e-mail: kornet@camk.edu.pl; mnr@camk.edu.pl \\ ${ }^{2}$ UCO/Lick Observatory, Department of Astronomy and Astrophysics, University of California, Santa Cruz,CA 95064, USA \\ e-mail: peter@ucolick.org
}

Received 26 July 2002 / Accepted 19 September 2002

\begin{abstract}
Formation of planets in the 47 UMa system is followed in an evolving protoplanetary disk composed of gas and solids. The evolution of the disk is calculated from an early stage, when all solids, assumed to be high-temperature silicates, are in the dust form, to the stage when most solids are locked in planetesimals. The simulation of planetary evolution starts with a solid embryo of $\sim 1$ Earth mass, and proceeds according to the core accretion - gas capture model. Orbital parameters are kept constant, and it is assumed that the environment of each planet is not perturbed by the second planet. It is found that conditions suitable for both planets to form within several Myr are easily created, and maintained throughout the formation time, in disks with $\alpha \approx 0.01$. In such disks, a planet of 2.6 Jupiter masses (the minimum for the inner planet of the 47 UMa system) may be formed at 2.1 AU from the star in $\sim 3 \mathrm{Myr}$, while a planet of 0.89 Jupiter masses (the minimum for the outer planet) may be formed at $3.95 \mathrm{AU}$ from the star in about the same time. The formation of planets is possible as a result of a significant enhancement of the surface density of solids between 1.0 and 4.0 AU, which results from the evolution of a disk with an initially uniform gas-to-dust ratio of 167 and an initial radius of $40 \mathrm{AU}$.
\end{abstract}

Key words. stars: planetary systems - stars: planetary systems: formation

\section{Introduction}

Planets in the mass range 0.1 to 10 Jupiter masses $\left(M_{J}\right)$, separated from their central stars by 0.04 to $5 \mathrm{AU}$, have been discovered around $\sim 100$ main-sequence stars with masses in the range 0.3-1.1 $M_{\odot}$. The general observed properties of these planets, several of which are in fact in planetary systems, are reviewed by Marcy et al. (2000), Perryman (2000), and Bodenheimer \& Lin (2002). In comparison to the solar system, many of these systems are unusual in two respects: they contain Jupiter-mass planets at close distances (down to $0.04 \mathrm{AU}$ ) from their star, and many of them are in orbits with substantial (0.4 to 0.7 ) eccentricity. However one particular system, belonging to the star 47 Ursae Majoris (47 UMa), stands out as being more solarsystem like than most of the other extrasolar planetary systems. This paper examines a possible mechanism for the origin of the two planets in the 47 UMa system.

The solar-type star 47 UMa has spectral type G0V, a mass of $1.03 M_{\odot}$, and a metallicity $[\mathrm{Fe} / \mathrm{H}]=-0.08$. One of the first extrasolar planets to be discovered was a companion to $47 \mathrm{UMa}$ (Butler \& Marcy 1996) at 2.09 AU, with a current minimum mass of $2.62 M_{J}$ and an eccentricity of 0.04. Later (Fischer et al. 2002) a second planet was discovered; current orbital

Send offprint requests to: K. Kornet, e-mail: kornet@camk . edu .pl parameters (Fischer 2002, private communication) give a semimajor axis of $3.95 \mathrm{AU}$, a minimum mass of $0.89 M_{J}$ and an eccentricity which is not well determined but is probably less than 0.1 . The mass ratio of the two planets (0.34) is close to that of Saturn/Jupiter (0.30), and the ratio of semimajor axes (1.90) is also close to that of Saturn/Jupiter (1.83). A dynamical analysis of the system (Laughlin et al. 2001) shows that if the two planets are in the same orbital plane, Earth-mass planets in the habitable zone would have stable orbits. However in the presence of the two fully formed giant planets, the formation of Earth-mass planets in the inner regions of the system is possible only interior to the habitable zone.

Three main theories have been proposed regarding the origin of planetary-mass objects. The first is dynamical fragmentation of a rotating collapsing protostar, the mechanism thought to be responsible for multiple stellar systems (reviewed by Bodenheimer et al. 2000a) and possibly the isolated planetarymass objects observed in the young cluster $\sigma$ Orionis (Zapatero Osorio et al. 2000). The second is gravitational instability in a disk (Kuiper 1951; Boss 2000; Boss et al. 2002), in which, on a few dynamical time scales, a gravitationally bound subcondensation forms in a disk that at some location has a Toomre $Q$ value on the order unity. The third mechanism, known as the core accretion - gas capture process, involves the relatively slow gradual accretion of small condensed particles in a disk, 
eventually resulting in a solid core of a few $M_{\oplus}$ which is able to gravitationally capture gas from the surrounding nebular disk (Safronov 1969; Perri \& Cameron 1974; Mizuno 1980). The strengths and weaknesses of the second and third processes are reviewed by Bodenheimer \& Lin (2002).

The formation of the inner giant planet in the $47 \mathrm{UMa}$ system has been studied by Bodenheimer et al. (2000b) under the assumption that it formed in situ by the core accretion - gas capture process. The evolutionary calculations they performed are based on the earlier work by Bodenheimer \& Pollack (1986), who assumed a constant solid accretion rate for the buildup of the core, and by Pollack et al. (1996) who employed more detailed physics, including a (non-constant) solid accretion rate calculated from three-body accretion cross sections. The aim of the calculation was to determine the disk properties needed to form the planet on a reasonable time scale at 2.1 AU. Two calculations with variable solid accretion rate were performed (their cases U1 and U2). The two most important parameters in such calculations are (1) the initial surface density of solid material $\Sigma_{\mathrm{s}}$, to which the formation time is highly sensitive, and (2) the grain opacity $\kappa_{\mathrm{g}}$ in the envelope of the protoplanet, to which the formation time is moderately sensitive (Pollack et al. 1996). In both cases presented by Bodenheimer et al. (2000b) the values of $\kappa_{\mathrm{g}}$ were based on interstellar grain properties as calculated, for example, by Pollack et al. (1985). In the temperature range 100-1500 K, those opacities are typically in the range $1-8 \mathrm{~cm}^{2} \mathrm{~g}^{-1}$. The values of $\Sigma_{\mathrm{s}}$ were set to 50 and $90 \mathrm{~g} \mathrm{~cm}^{-2}$ in the two calculations, and the formation times turned out to be 18.6 Myr and 1.9 Myr, respectively. The final solid core masses were 38 and $69 M_{\oplus}$, respectively, compared to a total assumed final mass of $2.5 M_{J}$ in both cases.

The lifetimes of disks around young stars, which constrain the formation times for giant planets, fall in the range 1-8 Myr (Haisch et al. 2001), with half of the disks in young clusters already gone after times of 3-4 Myr. The mass ratio of gas to solids in a solar-composition disk is expected to be about 200 at $2.1 \mathrm{AU}$, since the ice component is expected to be evaporated. Thus the total surface density of the disk, $\Sigma_{\text {total }}$, would have to be $1-2 \times 10^{4} \mathrm{~g} \mathrm{~cm}^{-2}$, in order for the planet to form in a reasonable time. If one examines the steadystate disk models of Bell et al. (1997) with viscosity parameter $\alpha=10^{-2}$, one sees that a disk with that high a $\Sigma_{\text {total }}$ at $2.1 \mathrm{AU}$ would have a temperature of about $1000 \mathrm{~K}$ (consistent with the evaporation of ices) and an accretion rate onto the star of $\dot{M} \approx 10^{-5} M_{\odot} \mathrm{yr}^{-1}$. Thus some difficulties with the model include (1) the required disk $\dot{M}$ is much higher than the values of $\sim 10^{-8} M_{\odot} \mathrm{yr}^{-1}$ in typical observed disks around young stars (Calvet et al. 2000), (2) such a disk is likely to be gravitationally unstable at larger radii (Bell et al. 1997) which could result in the formation of a much more massive planet at a distance of about $10 \mathrm{AU}$, and (3) $\Sigma_{\text {total }}$ is about 20 times that in the "minimum mass" solar nebula (Hayashi et al. 1985).

However a basic assumption of such disk models is that the ratio of gas to solids is constant at each radius as the disk evolves in time, except as modified by evaporation or condensation of solids. Here we consider alternate disk models, in which the evolution of $\Sigma_{\text {total }}$ and $\Sigma_{\mathrm{s}}$ is not necessarily coupled.
It has long been recognized that the solid particles in a disk evolve differently than the gas; for a review of the physical processes involved see Weidenschilling \& Cuzzi (1993). However global disk models in which the evolution of the solids and of the gas were followed consistently over timescales comparable to disk lifetimes (a few Myr) did not become available until Stepinski \& Valageas $(1996,1997)$ published some numerical solutions, based on a number of approximations in the physics. The typical result of such calculations was a decoupling of the evolution of the solid component from that of the gas, once the particle size had become large enough. At the end of a simulation, a typical disk had a region, say from 1 to $10 \mathrm{AU}$, where the ratio of surface densities of solid and gas was considerably higher than that suggested by solar composition, and also regions where solids were practically absent. For some disk models, however, all of the solid material accreted onto the star. Results for a larger region of parameter space for disk models were presented by Kornet et al. (2001), based on the methods of Stepinski \& Valageas $(1996,1997)$ but with further simplifications. These models start with a uniform, solar ratio of solids to gas, and evolve for $10^{7} \mathrm{yr}$, following the buildup of the initially small particles up to the size range $1-10 \mathrm{~km}$. The final stages of planet formation are not considered; a wide variety of possible distributions of solid material in evolved disks is found. The goal of the present paper is to investigate whether the formation, on a time scale of a few Myr, of both planets in the $47 \mathrm{UMa}$ system can be explained with reasonable disk models, based on the calculations of Kornet et al. (2001).

\section{Method of calculation}

\subsection{Disk models}

The method of calculation is described by Kornet et al. (2001). The gas component is modeled in one space dimension by an analytic solution to the viscous diffusion equation, which gives the surface density of the gas as a function of radius $r$ and time $t$ (Stepinski 1998). The viscosity is given by the usual $\alpha$ model. The temperature of the gas is calculated in the thin-disk approximation, assuming vertical thermal balance, according to Eqs. (2) through (6) in Stepinski (1998).

The main assumptions used in the calculation of the evolution of the solid component are (1) at each radius the particles are all assumed to have the same size, (2) there is only one component of dust, in this case corresponding to hightemperature silicates, which have an evaporation temperature of $1350 \mathrm{~K}$, (3) all collisions between particles lead to coagulation, (4) when the temperature exceeds the evaporation temperature, the solids are assumed to be in the form of vapor which evolves at the same radial velocity as the gas component, (5) when the disk temperature falls below the evaporation temperature at a given radius, all of the local vapor is assumed to condense immediately into grains with particle size $10^{-3} \mathrm{~cm}$, (6) the radial velocity of solid particles is determined by the effects of gas drag. The vertical thickness of the solid particle distribution at each radius is calculated and is evolved in time, so the effect of sedimentation of grains toward the midplane is 
taken into account. The evolution of solids does not affect the evolution of the density or the temperature of the gas.

The equations solved for the evolution of the solids include the continuity equation, the gas drag effect, coagulation and evaporation of particles. For the calculation of relative velocities at which coagulation proceeds, a turbulent model is assumed as described by Stepinski \& Valageas (1997). Those equations are solved numerically on a moving grid. Its outer boundary follows the motion of the outer edge of the solid disk. The ratio of radii at the inner and outer edges of the grid is kept constant. This ratio is chosen to be small enough for the dust velocities relative to the grid at the grid inner edge to be negative. In this way a free outflow boundary condition can be applied there. At the outer edge, since the dust velocity and the grid velocity are equal, no boundary condition is required. The grid points are equally spaced in log radius and their number is equal to 50 for every 3 orders of magnitude of the ratio of inner and outer radii. Further details of the code are given in Kornet et al. (2001).

The initial conditions can be parameterized by the quantities $m_{0}$ (the mass of the disk in $M_{\odot}$ ), and $j_{0}$ (the total angular momentum of the disk in units of $10^{52} \mathrm{~g} \mathrm{~cm}^{2} \mathrm{~s}^{-1}$ ). Once those parameters are chosen, the analytic solution of Stepinski (1998) gives the gas surface density as a function of radius at $t=0$. The ratio of the solid surface density to the gas surface density is initially set at the constant value of $6 \times 10^{-3}$, and the particle size is everywhere set to $10^{-3} \mathrm{~cm}$.

\subsection{Planet models}

The protoplanet consists of a solid core with a constant density of $3 \mathrm{~g} \mathrm{~cm}^{-3}$, appropriate for high-temperature silicates, and a gaseous envelope, both of which accrete mass according to the computational procedures described by Pollack et al. (1996) and Bodenheimer et al. (2000b); however certain simplifications are made. The basic assumptions are: (1) the protoplanet is surrounded by a disk with an initially uniform surface density $\Sigma_{\text {init,s }}$ of solid material, in the form of planetesimals. All planetesimals have the same size of $2 \mathrm{~km}$ (see below). The solid surface density $\Sigma_{\mathrm{s}}$ decreases with time as material accretes onto the protoplanet. (2) The protoplanet is assumed to be the dominant mass in the region of its feeding zone; accretion of solids onto other planetary embryos is not considered. Random velocities of the planetesimals are determined by only one planet in the feeding zone; thus they are expected to be small. The feeding zone is assumed to extend to 4 Hill sphere radii on either side of the protoplanet (Kary \& Lissauer 1994). (3) Diskplanet interactions and the resulting torques which could cause migration of the protoplanet through the disk are not considered. Planetesimals are assumed to be well mixed through the feeding zone at each time; thus the value of $\Sigma_{\mathrm{s}}$ is always uniform in space but usually decreasing with time. Planetesimals do not migrate into the feeding zone from outside, or vice versa. (4) Orbital parameters are kept constant, and it is assumed that the environment of each planet is not perturbed by the second planet.
Under these assumptions, the rate of accretion of solid material onto the protoplanet, taking into account the physical cross section of the growing planet as well as the gravitational enhancement factor, is given by the standard expression

$\dot{M}_{Z}=\pi R_{\mathrm{c}}^{2} \Sigma_{\mathrm{s}} \Omega F_{\mathrm{g}}$

where $\Omega$ is the orbital frequency, $R_{\mathrm{c}}$ is the effective capture radius of the protoplanet, and $F_{\mathrm{g}}$ is the gravitational enhancement factor. To simplify the calculation of $F_{\mathrm{g}}$ we modify this formula and use an expression given by Papaloizou \& Terquem (1999):

$\dot{M}_{Z}=C_{1} \pi R_{\mathrm{c}} R_{\mathrm{H}} \Sigma_{\mathrm{s}} \Omega$

where $R_{\mathrm{H}}$ is the Hill sphere radius. The value of $C_{1}$ given by Papaloizou \& Terquem (1999) is 81/32; we use a factor of 5. An expression of the form (2) has been shown to be consistent with the one of form (1) by Papaloizou \& Terquem (1999). Accretion rates from Eq. (2) are generally a factor 3 lower than those obtained from a combination of Eq. (1) and the calculations of $F_{\mathrm{g}}$ given by Greenzweig \& Lissauer (1992), which were used in the calculations of Pollack et al (1996) and Bodenheimer et al. (2000b).

The calculation of $R_{\mathrm{c}}$, the effective capture radius, takes into account the capture of planetesimals in the gaseous envelope. The procedure for taking into account the interaction of planetesimals with the envelope is described by Pollack et al. (1996), based on the work by Podolak et al. (1988). In the present calculations an approximate fit is made to the results of Bodenheimer et al. (2000b), provided by Hubickyj (2001). It was shown by Pollack et al. (1996) that at least for the case of Jupiter forming at $5 \mathrm{AU}$, the total formation time is insensitive to the planetesimal size assumed, in the range $1-100 \mathrm{~km}$. For core masses less than $5 M_{\oplus}$ the value of $R_{\mathrm{c}}$ is simply the core radius; for larger core masses the ratio of $R_{\mathrm{c}}$ to the core radius increases to about a factor 5 .

The structure of the gaseous envelope is determined from the equations of mass conservation, hydrostatic equilibrium, energy generation from accretion of planetesimals and quasistatic contraction, and radiative or convective energy transport, as given in Bodenheimer \& Pollack (1986). To avoid excessively large temperature gradients (which induce numerical instabilities), the energy deposition arising from the planetesimals landing on the core is smoothed over a region of about one core radius in extent. The molecular opacity in the envelope is based on calculations by Alexander \& Ferguson (1994). The grain opacity at temperatures less than the evaporation temperature of the most refractory species, taken to be $1800 \mathrm{~K}$, is set at a constant value of $0.03 \mathrm{~cm}^{2} \mathrm{~g}^{-1}$. This value is a factor of 50-100 less than the opacities obtained for grains with interstellar properties (Pollack et al. 1994). The coagulation and settling of grains in the atmosphere of a protoplanet results in a substantial reduction of opacity as compared with the interstellar values; a preliminary calculation by Podolak (2002) shows that in one particular case the maximum grain opacity in the radiative region of a protoplanet is only $0.02 \mathrm{~cm}^{2} \mathrm{~g}^{-1}$. Thus the value assumed above may be considered to be a conservative upper limit and would tend to overestimate the formation time. It is known that the formation time of a planet decreases as the 

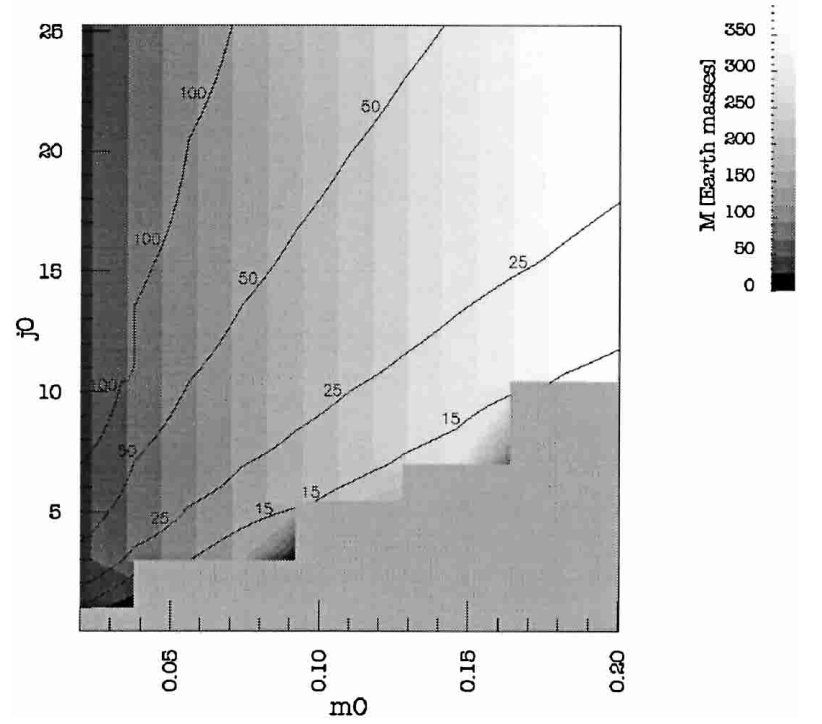

Fig. 1. Final mass and outer radius of the solid disk, as functions of the initial disk mass $m_{0}$ (in solar masses) and angular momentum $j_{0}$ (in units of $10^{52} \mathrm{~g} \mathrm{~cm}^{2} \mathrm{~s}^{-1}$ ). The contours give the outer radius in $\mathrm{AU}$, and the grey scale gives the mass in $M_{\oplus}$. The grey region at the lower right indicates disks in which the solid component has completely accreted onto the star.

opacity is reduced; for example Hubickyj et al. (2002) show that for a standard Jupiter model forming at 5 AU from the Sun, a reduction of a factor 50 in the grain opacity results in a reduction in the formation time by a factor of 2.2. The equation of state is non-ideal in the interior of the envelope; the tables of Saumon et al. (1995), are used, interpolated to a near-protosolar composition of $X=0.74, Y=0.243, Z=0.017$.

Boundary conditions at the inner edge of the envelope set the luminosity $L_{r}=0$ and the radius $r=R_{\text {core }}$, where $R_{\text {core }}$ is determined from the current core mass and the core density. At the outer edge temperature and density are given by the disk conditions at the appropriate distance from the star. The outer radius of the planet is assumed to fall at a modified accretion radius $R_{\mathrm{a}}$. Let the tidal, or Hill, radius be

$R_{\mathrm{H}}=a\left(\frac{M_{\mathrm{p}}}{3 M_{\star}}\right)^{1 / 3}$

where $a$ is the distance to the central star, $M_{\mathrm{p}}$ is the planet's mass, and $M_{\star}$ is the star's mass. Then $R_{\mathrm{a}}$ is given by

$R_{\mathrm{a}}=\frac{G M_{\mathrm{p}}}{c^{2}+\frac{G M_{\mathrm{p}}}{R_{\mathrm{H}}}}$

where $c$ is the sound speed in the nebula. In the limits of large and small $R_{\mathrm{H}}$, this expression reduces to the accretion radius and the tidal radius, respectively. The gas accretion rate is determined by the requirement that the outer radius of the protoplanet be close to $R_{\mathrm{a}}$, within a small tolerance. At every time step mass is added at the outer edge so that this requirement is satisfied.

The limiting gas accretion rate onto the planet is determined by the rate at which the nebula it is able to supply gas. Following Bodenheimer et al. (2000), we adopt for the latter
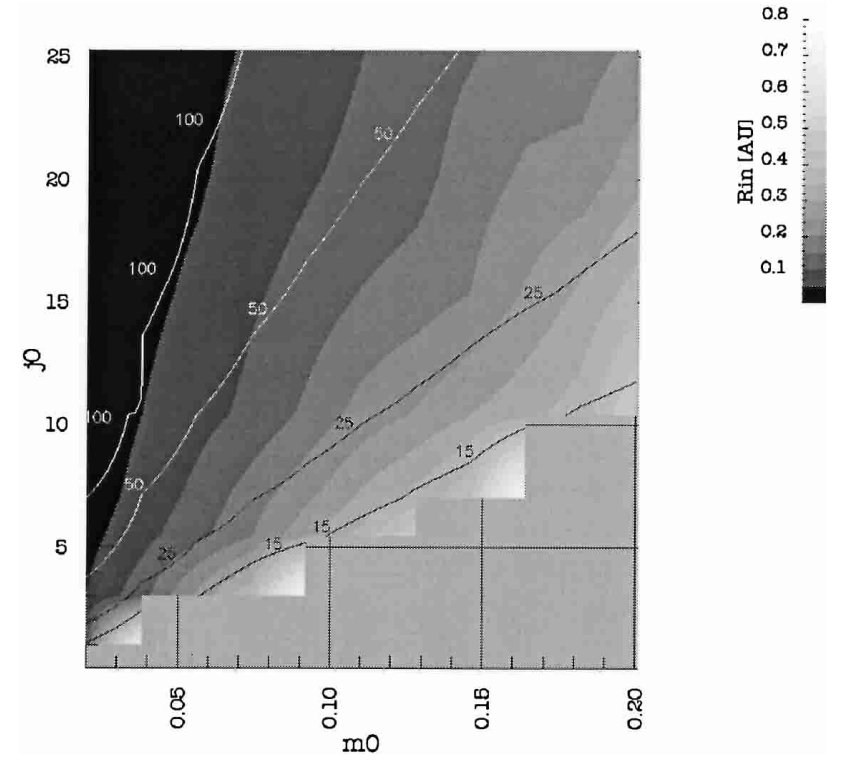

Fig. 2. Final inner radius and outer radius of the solid disk, as functions of the initial disk mass $m_{0}$ (in solar units) and angular momentum $j_{0}$ (in units of $10^{52} \mathrm{~g} \mathrm{~cm}^{2} \mathrm{~s}^{-1}$ ). The contours give the outer radius in $\mathrm{AU}$, and the grey scale gives the inner radius in AU. The grey region at the lower right indicates disks in which the solid component has completely accreted onto the star.

a value of $3 \times 10^{-8} M_{\odot} / \mathrm{yr}$ or $\approx 10^{-2} M_{\oplus} / \mathrm{yr}$, typical for the observed protoplanetary disks. Calculations are generally carried to the point where the limiting rate is reached. By that time the envelope mass has exceeded the core mass and the planet rapidly accretes gas up to its final mass, with only a relatively small change in the core mass. The formation time is in effect determined by the time needed to reach the crossover mass (envelope mass = core mass), so the calculation is stopped just beyond that point.

\section{Results}

We first discuss the general results of disk evolution as a function of $m_{0}$ and $j_{0}$. The dust and gas surface densities are evolved until either (1) the outer edge of the dust disk falls within $0.1 \mathrm{AU}$, in which case all the dust is assumed to have accreted onto the star, or (2) the total elapsed time is $10^{7} \mathrm{yr}$. In the second case, it usually occurs that the dust surface density distribution becomes constant in time, well before $10^{7} \mathrm{yr}$.

Figure 1 shows final disk properties as a function of $m_{0}$ and $j_{0}$ for a viscosity parameter $\alpha=10^{-2}$. The final outer radii of the dust disk are given by the contours, and the final solid mass is given by the greyscale. For a given $m_{0}$ and very low $j_{0}$ all of the solid material accretes onto the star. As $j_{0}$ is increased, the final solid mass increases rapidly, up to a saturation value, then remains constant with $j_{0}$. For a given value of $j_{0}$ the final mass of solids generally increases with $m_{0}$ except in the region in the $\left(m_{0}, j_{0}\right)$ plane where accretion of dust onto the star is significant. The grey area in Fig. 1 indicates the region in which all particles accrete onto the star (hereafter referred to as the dust accretion region). The steplike shape of its upper boundary is a consequence of the finite grid in $m_{0}$ (11 points) and $j_{0}$ 


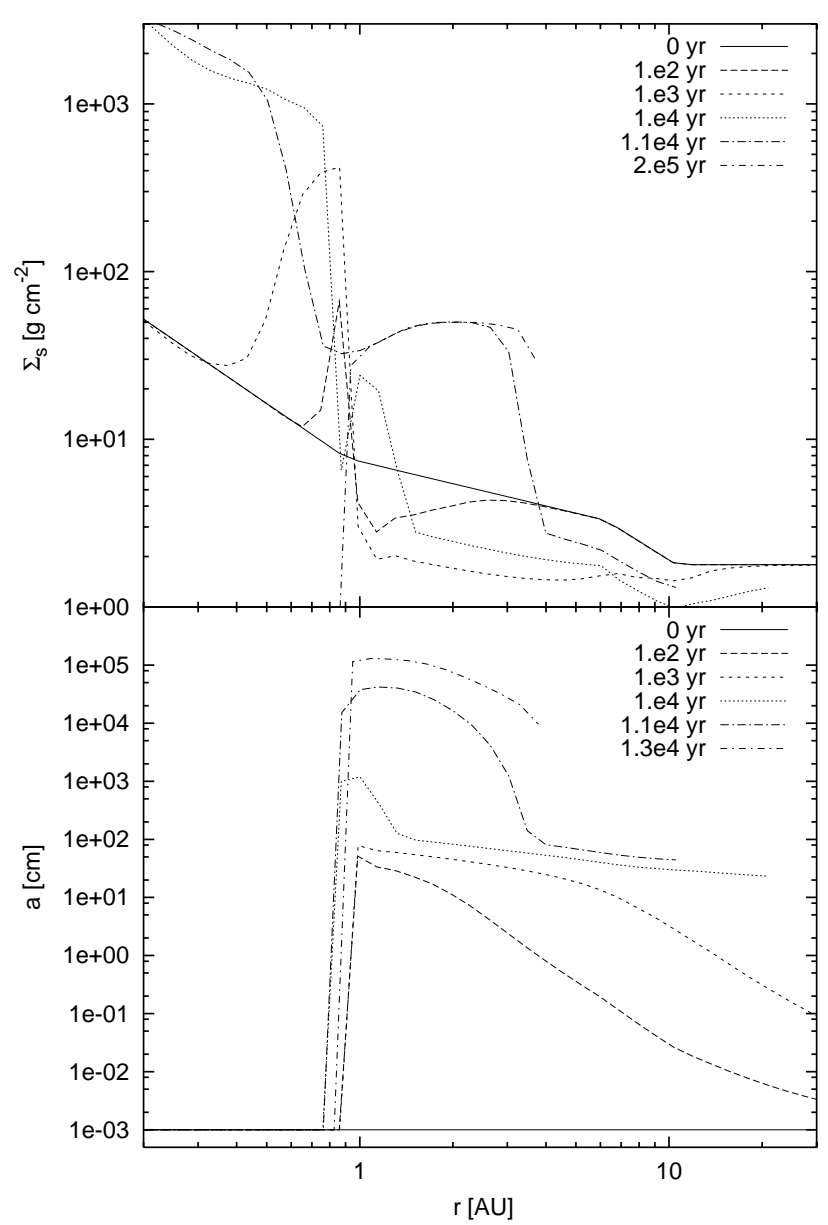

Fig. 3. Evolution of the solid component in the disk model used for planet formation in the $47 \mathrm{UMa}$ system $\left(m_{0}=0.164 M_{\odot}\right.$ and $j_{0}=7 \times 10^{52} \mathrm{~g} \mathrm{~cm}^{2} \mathrm{~s}^{-2}$ ). Top: surface density of solids in $\mathrm{g} \mathrm{cm}^{-2}$, as a function of distance from the star in $\mathrm{AU}$, at the times indicated. Bottom: particle radius in $\mathrm{cm}$ as a function of distance from the star in $\mathrm{AU}$, at the same times. Due to limited numerical resolution the location of the evaporation line is defined with the accuracy of $\sim 0.1 \mathrm{AU}$. After $1 \times 10^{4} \mathrm{yr}$ it stays practically constant.

(9 points). The boundary in a good approximation corresponds to disks in which at $t=0$ the evaporation radius is equal to the initial outer disk radius. The final outer dust radius generally increases with $j_{0}$, and decreases with $m_{0}$. The region in the $\left(m_{0}, j_{0}\right)$ plane which is most favorable for planet formation will be discussed below.

Figure 2, with a similar form to Fig. 1, shows again the outer radii of the final solid disk as contours, and the corresponding inner radii as greyscale. The general trend is that as the outer radius increases, the inner radius decreases. This effect occurs because as $m_{0}$ is decreased and $j_{0}$ is increased, the surface density of the gas disk, and therefore its temperature, decreases. As a result, the evaporation radius $\left(R_{\text {evap }}\right)$ is shifted inwards. Once the initial $R_{\text {evap }}$ is chosen, it can only move inward during the evolution. We find that the final inner radius of the dust disk is determined by the position of $R_{\text {evap }}$ at the particular time when the particles just outside $R_{\text {evap }}$ become large enough so they no longer migrate inward because of gas drag. In initially cooler disks, $R_{\text {evap }}$ at this moment is located closer

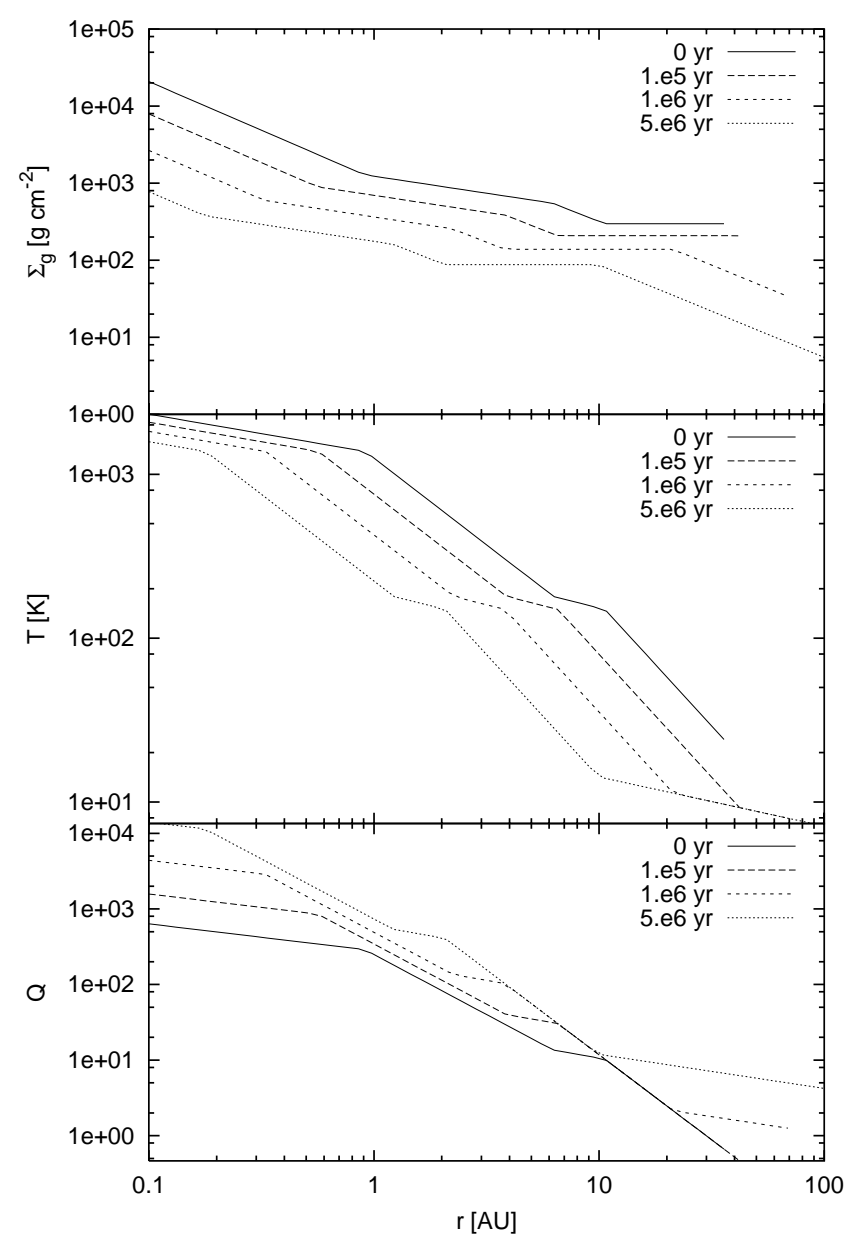

Fig. 4. Evolution of the gas component in the particular disk model used for planet formation in the $47 \mathrm{UMa}$ system. Top: surface density of the gas in $\mathrm{g} \mathrm{cm}^{-2}$, as a function of distance from the star in $\mathrm{AU}$, at the times indicated. Central frame: temperature in $\mathrm{K}$ as a function of distance from the star in $\mathrm{AU}$, at the same times. Bottom: Toomre $Q$ parameter as a function of distance from the star in $\mathrm{AU}$, at the same times.

to the star. Note that just outside the boundary of the dust accretion region in the $\left(m_{0}, j_{0}\right)$ plane, the final solid disks are rather compact, with the inner radius not much different from the outer radius. The presence of a compact disk tends to give a solid surface density high enough to allow the formation of a planet at relatively small distances (inside $5 \mathrm{AU}$ ) from the star.

The various disk models were examined to determine which one had the highest solid surface density at 2.1 AU at the final time. The parameters are $m_{0}=0.164$ and $j_{0}=7$, which places the model just above the boundary of the dust accretion region. The properties of the dust disk as a function of radius at different times are shown in Fig. 3. The plot shows both the solid surface density and the particle size. Initially the outer radius is about $40 \mathrm{AU}$ and the evaporation radius is at $0.9 \mathrm{AU}$. The solid surface density varies from about $10 \mathrm{~g} \mathrm{~cm}^{-2}$ at the evaporation radius to about $2 \mathrm{~g} \mathrm{~cm}^{-2}$ at the outer radius. Initially the particle sizes are all $10^{-3} \mathrm{~cm}$. As a function of time, outside the evaporation radius, the particle size increases, more slowly in the outer region because of the lower densities and collision rates. As the particle size increases, the vertical thickness of the 


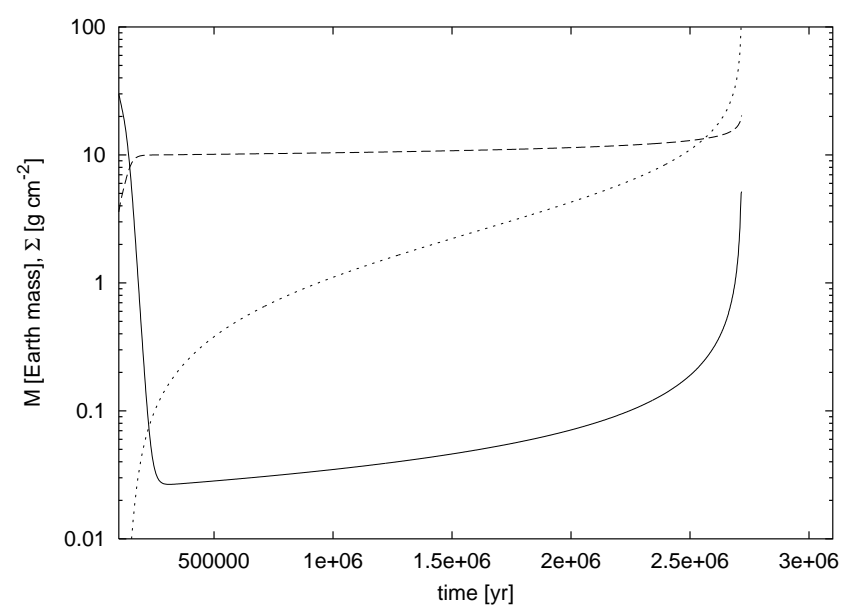

Fig. 5. Formation phase of a giant planet at 2.1 AU from the star with an assumed initial solid surface density of $50 \mathrm{~g} \mathrm{~cm}^{-2}$. Dashed, dotted, and solid lines indicate, respectively, core mass in $M_{\oplus}$, envelope mass in $M_{\oplus}$, and solid surface density in $\mathrm{g} \mathrm{cm}^{-2}$ remaining in the disk at the location of the planet, all as a function of time in years (counted from the beginning of the disk evolution).

dust disk decreases. At $100 \mathrm{yr}$ some of the solid material has migrated inside the evaporation radius, and the sharp maximum in $\Sigma_{\mathrm{s}}$ actually is composed of vapor. The region just outside the evaporation radius is depleted in solids. At 1000 years the maximum $\Sigma_{\mathrm{s}}$ in the vapor region is higher and has been somewhat smoothed by the viscosity (in this region the vapor is directly coupled to the gas). The region of depletion outside the evaporation radius is larger. At $10000 \mathrm{yr}$ the inner maximum in the vapor region has been completely smoothed out, and another maximum with $\Sigma_{\mathrm{s}}=25 \mathrm{~g} \mathrm{~cm}^{-2}$ has formed, just outside the evaporation radius, at $1 \mathrm{AU}$. This region is populated by particles that have migrated from the outer regions of the disk, but have grown to large enough size $(20 \mathrm{~m})$ so that they no longer migrate. A short time later $\left(1.1 \times 10^{4} \mathrm{yr}\right)$ this peak becomes somewhat higher and spreads outward in radius, while the outer part of the disk, beyond $4 \mathrm{AU}$, is strongly depleted as compared with the initial particle density. At this time the outer radius of the solid disk has decreased to about $10 \mathrm{AU}$. Beyond this time the region of high solid surface density between 1 and 3 AU does not evolve, because the particles are large enough so they do not migrate; the particles just increase in size. The region of high density increases somewhat in radius as particles from the outer regions migrate into it. At $2 \times 10^{5} \mathrm{yr}$ the evolution of $\Sigma_{\mathrm{s}}$ stops with an inner radius of $0.9 \mathrm{AU}$ and an outer radius of $4 \mathrm{AU}$. The values of $\Sigma_{\mathrm{s}}$ at $2.1 \mathrm{AU}$ and $3.95 \mathrm{AU}$ are 50 and $\approx 15 \mathrm{~g} \mathrm{~cm}^{-2}$, respectively. The outer value is approximate because it falls very close to the outer edge of the solid disk. At this time the typical particle size is $1 \mathrm{~km}$; at later times the particle size would tend to increase even further, but the model for the solid accretion is no longer valid because it does not include gravitational effects. Note also that $a(r)$ approaches a constant value, consistent with the assumption of constant planetesimal size that is made in Sect. 2.2.

Figure 4 shows the evolution of the gas in the particular disk shown in Fig. 3. The surface density $\Sigma_{\mathrm{g}}$ generally decreases in time and the disk expands in radius, as would be expected for a

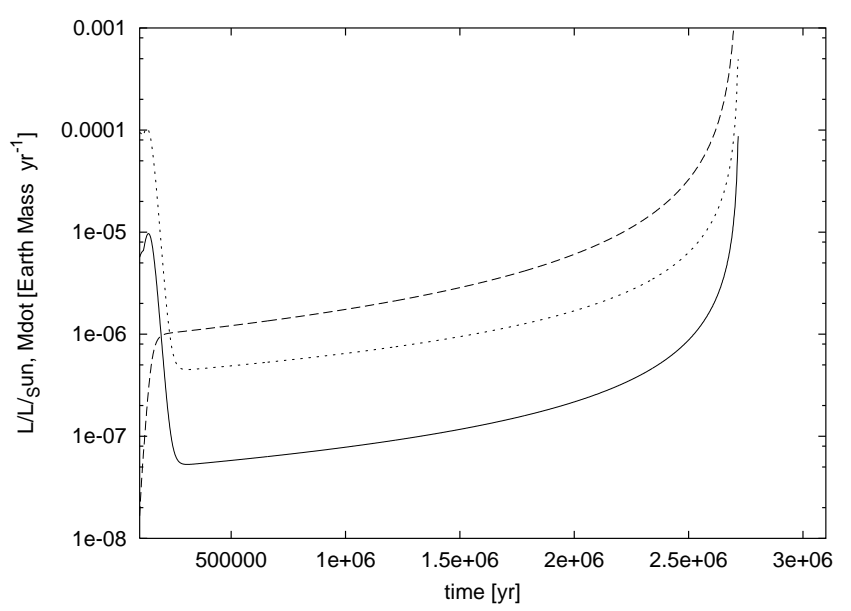

Fig. 6. For the same model as in Fig. 5, dotted, dashed, and solid curves indicate, respectively, the accretion rate of solid material onto the core in $M_{\oplus} \mathrm{yr}^{-1}$, the accretion rate of gas into the envelope in $M_{\oplus} \mathrm{yr}^{-1}$, and the radiated luminosity of the protoplanet, in $L_{\odot}$, all as a function of time in years (counted from the beginning of the disk evolution).

standard accretion disk. The mass of the gas decreases to 0.15 , 0.11 , and $0.09 M_{\odot}$ at times of $1 \times 10^{5}, 1 \times 10^{6}$, and $5 \times 10^{6} \mathrm{yr}$, respectively. On the temperature plot, the evaporation radius is always the first point (in radius) where the slope changes. It moves inward from about 0.9 AU to 0.2 AU. The slope changes correspond to changes in the dust opacity, which is assumed to vary as a power law in temperature, with different exponents in different regions of temperature (Stepinski 1998). The lower portion of Fig. 4 shows the Toomre $Q$ stability parameter. For values above $\approx 1$ the disk would not be expected to form planets by the gravitational instability mechanism. The initial gas disk is in fact gravitationally unstable outside $30 \mathrm{AU}$. The mass in the unstable region is about $0.1 M_{\odot}$, so there is at least a possibility, untested by detailed numerical simulations, that a planet could form rapidly by gravitational instability in the very outer region. At later times the disk becomes increasingly stable, and it is always highly stable in the region from 2 to $5 \mathrm{AU}$.

Planet formation at 2.1 AU is assumed to start when the particle size reaches $2 \mathrm{~km}$, which occurs at a time of $2.1 \times 10^{4} \mathrm{yr}$ (this time corresponds to a definite upper limit of the applicability of the disk evolution code), This choice is somewhat arbitrary, but both the early evolution of planetesimals and assembly of the protoplanetary core proceed so rapidly, that their time scale is at least one order of magnitude shorter then the core and envelope accretion time scale. The $\Sigma_{\mathrm{s}}$ has reached a value of 50 and does not change in time. Assuming that there is one dominant planetary core, Eq. (2) is integrated in time, using appropriate parameters for $2.1 \mathrm{AU}$, starting with a core mass of $10^{17} \mathrm{~g}$ and ending at $1 M_{\oplus}$. The calculated time is $5 \times 10^{4} \mathrm{yr}$, so the starting time for the full planetary formation calculation is at $7 \times 10^{4} \mathrm{yr}$. The surface boundary conditions for the forming planet are taken from the disk model at that time, which has a mass density $\rho=7 \times 10^{-11} \mathrm{~g} \mathrm{~cm}^{-3}$ and a temperature $T=400 \mathrm{~K}$ at $2.1 \mathrm{AU}$. At later times $(\approx 2 \mathrm{Myr})$ these surface values decrease to $\rho=4.7 \times 10^{-11} \mathrm{~g} \mathrm{~cm}^{-3}$ and $T=170 \mathrm{~K}$. 


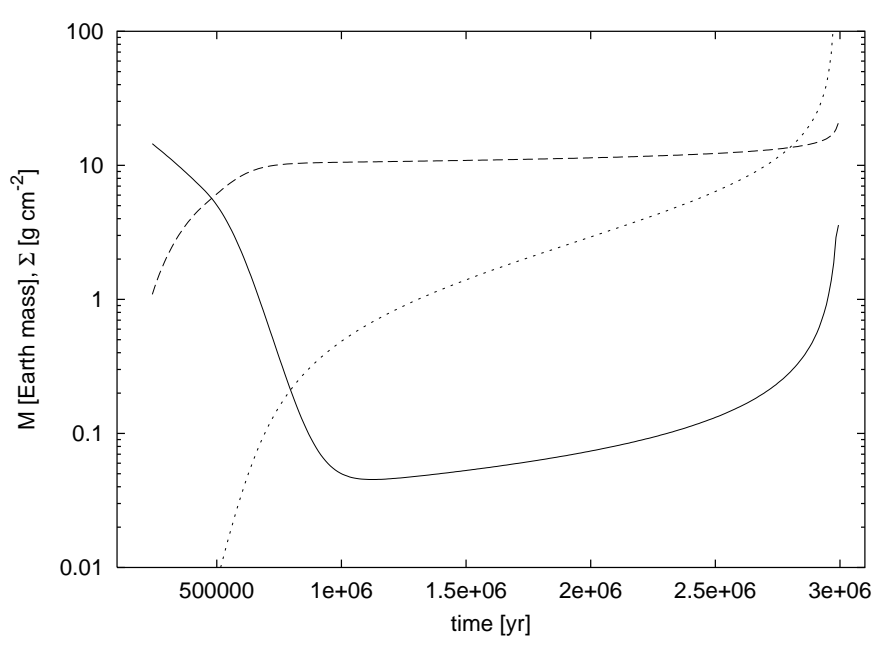

Fig. 7. Formation phase of a giant planet at $3.95 \mathrm{AU}$ from the star with an assumed initial solid surface density of $15 \mathrm{~g} \mathrm{~cm}^{-2}$. Dashed, dotted, and solid lines indicate, respectively, core mass in $M_{\oplus}$, envelope mass in $M_{\oplus}$, and solid surface density in $\mathrm{g} \mathrm{cm}^{-2}$ remaining in the disk at the location of the planet, all as a function of time in years (counted from the beginning of the disk evolution).

The results are shown in Figs. 5 and 6. Figure 5 shows the solid core mass, the gaseous envelope mass, and the solid surface density in the disk as a function of time. Figure 6 shows the rate of increase of core mass, the rate of increase of envelope mass, and the radiated luminosity (in solar units) as a function of time. The evolution is divided into three phases. Phase 1 is characterized by a fairly rapid increase in the core mass during the period when the envelope mass is negligible. The solid accretion rate peaks at $10^{-4} M_{\oplus} \mathrm{yr}^{-1}$, then declines as the core accretes most of the solid mass within the feeding zone. The rapid drop in $\Sigma_{\mathrm{s}}$ during this phase is evident in Fig. 5. The core mass builds up to $10 M_{\oplus}$ on a time scale of $10^{5} \mathrm{yr}$. The luminosity, which is provided by accretion of planetesimals onto the core, peaks at about $10^{-5} L_{\odot}$. The accretion rate of the envelope is small compared with that of the core, but is rapidly increasing. Phase 2 starts when the core mass has leveled off and the accretion rates of core and envelope become equal at $10^{-6} M_{\oplus} \mathrm{yr}^{-1}$, which occurs at a total elapsed time of $2.3 \times 10^{5} \mathrm{yr}$. During this phase, which determines the overall formation time scale, the envelope accretion rate is a factor 2-3 larger than that of the core, so the envelope mass builds up more rapidly than that of the core. The luminosity remains at a low value of $10^{-7}-10^{-6} L_{\odot}$. Phase 3 begins at $t=2.6 \times 10^{6} \mathrm{yr}$ when crossover mass is reached, with envelope mass equal to core mass (13.3 $M_{\oplus}$ in this case), and proceeds with a rapidly increasing accretion rate for the envelope. The plot cuts off at $100 M_{\oplus}$, but the calculation was continued until the (minimum) mass of $47 \mathrm{UMa}$ b, $2.62 M_{J}$, was reached. The formation time to final mass is $2.7 \mathrm{Myr}$, and the final core mass is $21 M_{\oplus}$. During Phase 3, the luminosity is provided primarily by rapid contraction of the envelope itself, and it rapidly increases to a second peak of $\approx 10^{-2} L_{\odot}$ (not shown; see Bodenheimer et al. 2000).

Planet formation at 3.95 $\mathrm{AU}$ is assumed to start when the particle size reaches $2 \mathrm{~km}$, which occurs at a time of $4 \times 10^{4} \mathrm{yr}$.

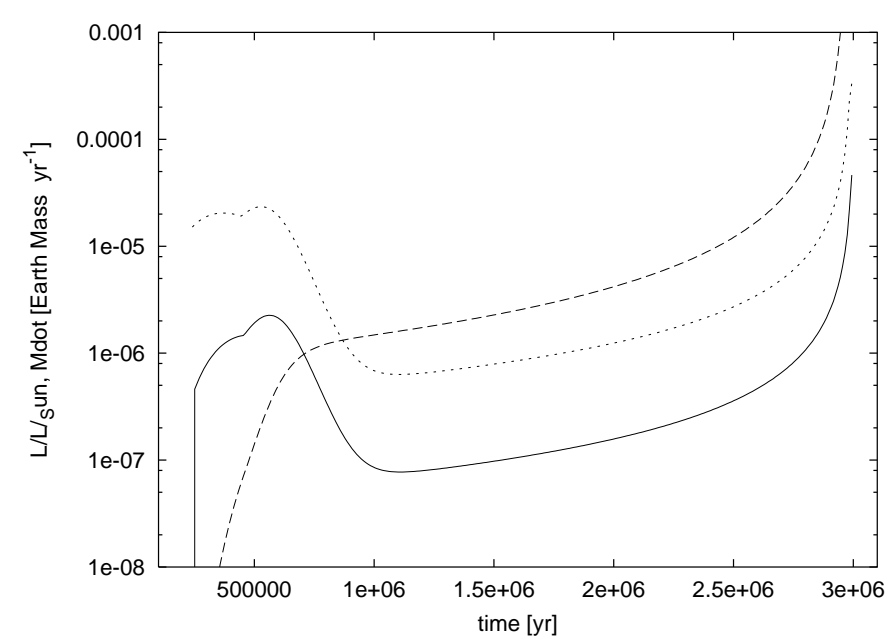

Fig. 8. For the same model as in Fig. 7, dotted, dashed, and solid curves indicate, respectively, the accretion rate of solid material onto the core in $M_{\oplus} \mathrm{yr}^{-1}$, the accretion rate of gas into the envelope in $M_{\oplus} \mathrm{yr}^{-1}$, and the radiated luminosity of the protoplanet, in $L_{\odot}$, all as a function of time in years (counted from the beginning of the disk evolution).

The $\Sigma_{\mathrm{s}}$ has reached a value of $\approx 15$ and does not change in time. The buildup time from $2 \mathrm{~km}$ planetesimals to a core of $1 M_{\oplus}$ is calculated to be $2 \times 10^{5} \mathrm{yr}$, so the starting time for the full planetary formation calculation is $2.4 \times 10^{5} \mathrm{yr}$. The surface boundary conditions for the forming planet are taken from the disk model at that time, which has a mass density $\rho=2 \times 10^{-11} \mathrm{~g} \mathrm{~cm}^{-3}$ and a temperature $T=170 \mathrm{~K}$ at $3.95 \mathrm{AU}$. At $2 \times 10^{6} \mathrm{yr}$ these values have decreased to $\rho=1.6 \times 10^{-11} \mathrm{~g} \mathrm{~cm}^{-3}$ and $T=111 \mathrm{~K}$.

The planet at 3.95 AU is assumed to be formed independently of the one at 2.1 AU; in fact their feeding zones do not overlap. This assumption is somewhat restrictive as in principle another protoplanetary core(s) could form between 2.1 and 3.95 AU. However we just want to demonstrate that the in situ formation of the $47 \mathrm{UMa}$ system is possible and therefore we do not consider any other evolutionary scenarios. The results are shown in Figs. 7 and 8, which show the same quantities as in Figs. 5 and 6, respectively. During Phase 1, the core accretion rate increases to a maximum of $2.5 \times 10^{-4} M_{\oplus} \mathrm{yr}^{-1}$ and then declines as $\Sigma_{\mathrm{s}}$ in the disk is depleted. The core mass builds up to $10 M_{\oplus}$ on a time scale of $3 \times 10^{5} \mathrm{yr}$. The luminosity during Phase 1 peaks at about $2.5 \times 10^{-6} L_{\odot}$, somewhat lower than that for the planet at 2.1 AU. Phase 1 ends when the accretion rate of the envelope, previously low, equals that of the core, at $8.5 \times 10^{5} \mathrm{yr}$, a factor 3.7 longer than the corresponding time for the planet at 2.1 AU. Phase 2, however, is very similar in the two cases with regard to time scale, accretion rates, and luminosity. The crossover mass of $13 M_{\oplus}$ is reached at $2.8 \times 10^{6} \mathrm{yr}$, slightly later than that for the planet at $2.1 \mathrm{AU}$. The calculation was continued until the minimum mass of $47 \mathrm{UMa} c, 0.89 M_{J}$, was reached after a time of $3.0 \times 10^{6} \mathrm{yr}$, only $3 \times 10^{5} \mathrm{yr}$ longer than that for the inner planet. The final core mass has increased slightly since crossover to $16 M_{\oplus}$. 


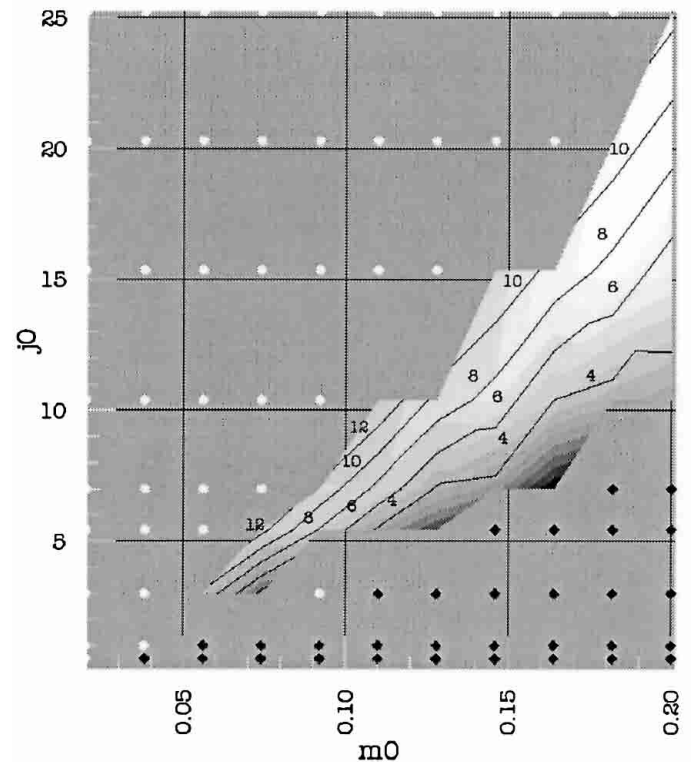

Fig. 9. As functions of the initial disk mass $m_{0}$ (in solar masses) and angular momentum $j_{0}$ in units of $10^{52} \mathrm{~g} \mathrm{~cm}^{2} \mathrm{~s}^{-1}$, the contours and grey scale, respectively, give the inner and outer radius of the region around the central star where giant planet formation is possible in a maximum of 3 Myr. White circles indicate disk models where the solid surface density is everywhere below the critical value for planet formation. Diamonds indicate disks in which all of the solid material accretes onto the star. The disk viscosity parameter $\alpha=1 \times 10^{-2}$.

\section{Conclusions}

These numerical results allow us to reach the following conclusions: (1) there exists a disk model which allows the formation of both of the planets in the 47 UMa system in about $3 \mathrm{Myr}$ at their present distances from the star. (2) The initial disk may be significantly less massive than the one required by Bodenheimer et al. (2000). At 2.1 AU their gas density had to be as high as $2.1 \times 10^{4} \mathrm{~g} \mathrm{~cm}^{-2}$, while the disk used in the present calculation had $\Sigma_{\mathrm{g}}=10^{3} \mathrm{~g} \mathrm{~cm}^{-2}$ at $2.1 \mathrm{AU}$ at the beginning of the disk evolution. (3) The solid cores of both planets are relatively small, 21 and $16 M_{\oplus}$ for the inner and outer planet, respectively. In comparison, the core mass for the inner planet in model U2 of Bodenheimer et al. (2000), which formed in about $2 \mathrm{Myr}$, was $69 M_{\oplus}$. (4) The planet at $2.1 \mathrm{AU}$ formed in a much shorter time (2.7 Myr vs. 18.6 Myr) than that with the same assumed solid surface density in the the calculations of Bodenheimer et al. (2000b). The main reason is that the grain opacity in the present calculation is up to a factor 100 lower than that assumed by Bodenheimer et al. (2000b). That effect is known to lead to shorter formation times. A test calculation for the inner planet was made in which the grain opacity was reset to interstellar values, as used by Bodenheimer et al. (2000b), all other effects remaining the same. The formation time turned out to be $1.2 \times 10^{7} \mathrm{yr}$, more than a factor 4 longer than the 2.7 Myr obtained with the reduced opacity, but about $30 \%$ shorter than in Bodenheimer et al. (2000b). The latter difference is explained by the fact that the earlier calculation had different surface boundary conditions, a different procedure for
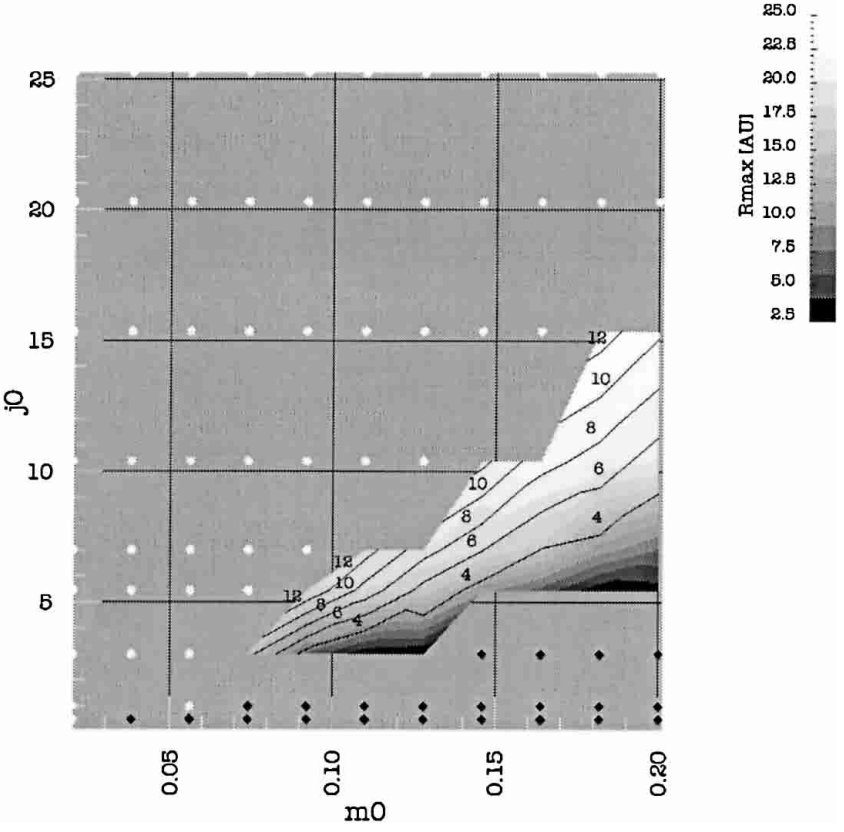

Fig. 10. The symbols and grey scale have the same meaning as in Fig. 9 , for $\alpha=1 \times 10^{-3}$.

calculating $\dot{M}_{Z}$, and a higher core density $\left(5 \mathrm{~g} \mathrm{~cm}^{-3}\right)$. (5) In the presence of the fully formed planet at $2.1 \mathrm{AU}$, there is still some solid material left in the disk between 1 and $1.5 \mathrm{AU}$, with $\Sigma_{\mathrm{s}} \approx 40 \mathrm{~g} \mathrm{~cm}^{-2}$ and a mass of about $6 M_{\oplus}$. Formation of another giant planet in this region is not possible; a numerical calculation shows that the formation time would be much longer than the lifetime of the gas disk. The minimum solid surface density required to form Jupiter size planet in this region in $3 \mathrm{Myr}$ is $\sim 100 \mathrm{~g} \mathrm{~cm}^{-2}$ (see below).

These results lead to the question: in what kinds of disks is it possible to form Jupiter size planets on a 3 Myr time scale? We calculated approximate planet formation models, fitted to the results of Sect. 3, to determine, at various distances from a $1 M_{\odot}$ star, the minimum solid surface density $\Sigma_{\mathrm{s} \text {,min }}$ needed to form a giant planet in $3 \mathrm{Myr}$. The results for $\Sigma_{\mathrm{s}, \min }$ range from $100 \mathrm{~g} \mathrm{~cm}^{-2}$ at $1.0 \mathrm{AU}$ to $9 \mathrm{~g} \mathrm{~cm}^{-2}$ at $5 \mathrm{AU}$ to a minimum of $3 \mathrm{~g} \mathrm{~cm}^{-2}$ at $15 \mathrm{AU}$. Then they increase slowly outward to $4.6 \mathrm{~g} \mathrm{~cm}^{-2}$ at $50 \mathrm{AU}$. This result can be explained as follows. At small $R$ the protoplanet spends most of the evolutionary time in phase 2 . The time scale for phase 2 depends strongly on the core mass (see Pollack et al. 1996 for a detailed explanation), so that in order to form the planet in a given time, say $3 \mathrm{Myr}$, the core mass must exceed some critical mass $M_{\text {crit }} \sim 15 M_{\oplus}$. Thus the necessary condition to form the planet at a given location is that the isolation mass, $M_{\text {iso }}$ is not smaller then $M_{\text {crit }}$. According to Pollack et al. (1996)

$M_{\text {iso }}=C_{1}\left(R^{2} \Sigma_{\mathrm{s}}\right)^{3 / 2}$.

Setting $M_{\text {iso }}=M_{\text {crit }}=$ const we recover the rapid increase of $\Sigma_{\mathrm{s}, \min }$ toward the center of the disk as observed in numerical results. On the other hand, at large $R$ the evolutionary time scale is determined by the length of phase 1 in which the core is assembled. Here, the necessary condition for the planet to form 
within a prescribed time is that the mass of the core reaches $M_{\text {crit }}$. Setting $M_{\text {crit }}=$ const and employing formula (2) we get

$\Sigma_{\mathrm{s}, \min } \sim R^{1 / 2}$,

again in a rough agreement with the numerical results.

We then examined all the disk models shown in Figs. 1 and 2 to find the range of distances $R_{\min } \leq R \leq R_{\max }$ at which the final $\Sigma_{\mathrm{s}}$ exceeds the local $\Sigma_{\mathrm{s}, \min }$. Figure 9 shows the results for disk models with $\alpha=10^{-2}$, as a function of the fundamental disk parameters $m_{0}$ and $j_{0}$. The grey scale gives the values of $R_{\max }$ and the contours give the values of $R_{\min }$. Note that the region of possible planet formation at less than $5 \mathrm{AU}$ is very limited. In fact we can estimate, consistent with this model, that the minimum radius at which giant planet formation is possible is about $2 \mathrm{AU}$. Also, the maximum radius is estimated at $23 \mathrm{AU}$. In general, if $R_{\min }$ is small, $R_{\max }$ is also relatively small. In models marked by a white circle, there is solid material present at the end of the evolution, but $\Sigma_{\mathrm{s}}$ is everywhere too low to form planets. In models marked by a diamond, all of the solid material accretes onto the star. Note that the disk models do not include ice grains, which will be included in future calculations. However the minimum radius for planet formation is not expected to change if ice is included.

Figure 10 shows the same results as Fig. 9 except for a disk viscosity parameter $\alpha=10^{-3}$. The minimum radius for planet formation in this case is somewhat smaller, about $1 \mathrm{AU}$, and the maximum is somewhat larger, about 25 AU. The main effect of the reduction in $\alpha$ is a more extended region in which the dust survives (Stepinski \& Valageas 1997). The inner radii of the final dust disk are smaller because the lower- $\alpha$ disks have lower temperatures as a result of a smaller energy generation rate by viscosity at a given surface density. Thus the evaporation radius tends to be at smaller distances. On the other hand, the outer radii of the dust disks are larger because the inward drift of particles is slower. Another effect of reducing $\alpha$ is to reduce the maximum $j_{0}$ for which planet formation is possible. Generally as $j_{0}$ is increased for a given $m_{0}$, the gas and dust disks become more extended and therefore have lower average surface density. For the same disk parameters except for a lower $\alpha$, the dust disk is more extended, so the $\Sigma_{\mathrm{s}}$ falls everywhere below the critical value at smaller $j_{0}$.

In summary, the planets in the 47 UMa system can be explained by the core accretion - gas capture process in a disk with reasonable parameters (mass $0.16 M_{\odot}$ and outer initial radius $40 \mathrm{AU}$ ) in which the dust component consists of hightemperature silicates. The inclusion of ice grains in the model would probably make little difference in the planet formation process: a corresponding disk model calculated with only ice grains initially present resulted in the accretion of all of the ice onto the star. The planets can reach their observed minimum masses in 3 Myr. The main factor enabling their formation is that the surface density of solids in the region $2-4 \mathrm{AU}$ is considerably higher than in the 'minimum mass' solar nebula.

Migration of the planets as a result of gravitational interactions with the disk was not included. However migration is still a possibility since with slightly changed disk parameters the planets might be formed at much larger distances from the star (note that in the present model the inner planet is located at about the minimum distance from the star where it is possible to form a giant planet in an $\alpha=10^{-2}$ disk).

Future improvements of the model should include (1) interactions between different types of solid particles, (2) particle models in which a range of particle sizes at each position in the disk is considered, (3) improvements in the dust opacity in the envelopes of the protoplanets, which influences the time scale of Phase 2, and (4) a better description of the boundary between the envelope of the planet and the disk; in particular, the presence of the secondary (circumplanetary) disk described by Ciecielag et al. (2000) and D'Angelo et al. (2002). Finally, the effect of increased or reduced metal abundance on the region of parameter space in which planet formation is possible should also be investigated.

Acknowledgements. This work was supported in part through NASA grants NAG5-9661 and NAG5-9526 from the Origins of Solar Systems Program, through Polish Committee for Scientific Research grant 2P03D01419 and by the European Commission through RTN grant No. HPRN-CT-2002-00308. We thank Debra Fischer for providing updated observational data on the properties of the $47 \mathrm{UMa}$ system.

\section{References}

Alexander, D. R., \& Ferguson, J. W. 1994, ApJ, 437, 879

Bell, K. R., Cassen, P. M., Klahr, H. H., \& Henning, Th. 1997, ApJ, 486, 372

Bodenheimer, P., \& Pollack, J. B. 1986, Icarus, 67, 391

Bodenheimer, P., Burkert, A., Klein, R. I., \& Boss, A. P. 2000a, in Protostars and Planets IV, ed. V. Mannings, A. P. Boss, \& S. Russell (Univ. of Arizona Press, Tucson), 675

Bodenheimer, P., Hubickyj, O., \& Lissauer J. J. 2000b, Icarus, 143, 2

Bodenheimer, P., \& Lin, D. N. C. 2002, Annu. Rev. Earth Planet. Sci., 30,113

Boss, A. P. 2000, ApJ, 536, L101

Boss, A. P., Wetherill, G. W., \& Haghighipour, N. 2002, Icarus, 156, 291

Butler, R. P., \& Marcy, G. W. 1996, ApJ, 464, L153

Calvet, N., Hartmann, L. W., \& Strom, S. E. 2000, in Protostars and Planets IV, ed. V. Mannings, A. P. Boss, \& S. Russell (Univ. of Arizona Press, Tucson), 377

Ciecielag, P., Plewa, T., \& Różyczka, M. 2000, Astron. Nachr., 321, 171

D’Angelo, G., Henning, Th., \& Kley, W. 2002, A\&A 385, 647

Fischer, D. A. 2002, private communication

Fischer, D. A., Marcy, G. W., Butler, R. P., Laughlin, G., \& Vogt, S. S. 2002, ApJ, 564, 1028

Greenzweig, Y., \& Lissauer, J. J. 1992, Icarus, 100, 440

Haisch, K. E., Jr, Lada, E. A., \& Lada, C. J. 2001, ApJ, 553, L153

Hayashi, C., Nazawa, K., \& Nakagawa, Y. 1985, in Protostars and Planets II, ed. D. C. Black \& M. S. Matthews (Univ. of Arizona Press, Tucson), 1100

Hubickyj, O. 2001, private communication

Hubickyj, O., Bodenheimer, P., \& Lissauer, J. J. 2002, in preparation

Kary, D. M., \& Lissauer, J. J. 1994, in Numerical Simulations in Astrophysics, ed. J. Franco, S. Lizano, L. Aguilar, \& E. Daltabuit (Cambridge Univ. Press, Cambridge), 364 
Kornet, K., Stepinski, T. F., \& Różyczka, M. 2001, A\&A, 378, 180

Kuiper, G. P. 1951, in Astrophysics, ed. J. A. Hynek (McGraw Hill, New York), 357

Laughlin, G., Chambers, J., \& Fischer D. 2001, Am. Astron. Soc. Meeting 199, abstract 03.08

Marcy, G. W., Cochran, W. D., \& Mayor, M. 2000, in Protostars and Planets IV, ed. V. Mannings, A. P. Boss, \& S. Russell (Univ. of Arizona Press, Tucson), 1285

Mizuno, H. 1980, Prog. Theor. Phys., 64, 544

Papaloizou, J. C. B., \& Terquem, C. 1999, ApJ, 521, 823

Perri, F., \& Cameron, A. G. W. 1974, Icarus, 22, 416

Perryman, M. A. C. 2000, Rep. Prog. Phys., 63, 1209

Podolak, M. 2002, in preparation

Podolak, M., Pollack, J. B., \& Reynolds, R. T. 1988, Icarus, 73, 163

Pollack, J. B., McKay, C., \& Christofferson, B. 1985, Icarus, 64, 471
Pollack, J. B., Hollenbach, D., Beckwith, S., et al. 1994, ApJ, 421, 615

Pollack, J. B., Hubickyj, O., Bodenheimer, P., et al. 1996, Icarus, 124, 62

Safronov, V. S. 1969, Evolution of the Protoplanetary Cloud and Formation of the Earth and Planets (Nauka Press, Moscow, in Russian). English translation: NASA-TT-F-677, 1972.

Saumon, D., Chabrier, G., \& van Horn, H. M. 1995, ApJS, 99, 713

Stepinski, T. F., \& Valageas, P. 1996, A\&A, 309, 301

Stepinski, T. F., \& Valageas, P. 1997, A\&A, 319, 1007

Stepinski, T. F. 1998, Icarus, 132, 100

Weidenschilling, S. J., \& Cuzzi, J. N. 1993, in Protostars and Planets III, ed. E. H. Levy, \& J. I. Lunine (Univ. of Arizona Press, Tucson), 1031

Zapatero Osorio, M. R., Bejar, V. J. S., Martin, E. L., et al. 2000, Science, 290, 103 